\title{
SEGURIDAD DE LA HISTEROSCOPIA OFICINAL EN UNA UNIDAD DE FERTILIDAD. BOGOTÁ, COLOMBIA, 2011-2016. COHORTE RETROSPECTIVA
}

\section{Safety of office hysteroscopy in a fertillity unit. Bogota, Colombia, 2011-2016. Retrospective cohort}

Ivonne Jeannette Díaz-Yamal, $\mathrm{MD}^{1}$; Angélica María Uscátegui-Diago, $\mathrm{MD}^{2}$;

Jimmy Castañeda-Castañeda, $M D^{3}$; Carlos Andrés Fandiño-Rodríguez, $M^{3}{ }^{3}$; Jaime Edward Villamil-Pérez, $M D^{4}$; Fernando Gómez-Corredor, $M D^{5}$; Mario Luis Pájaro-Corredor, $M^{2}$

Recibido: septiembre 7/16 - Aceptado: agosto 22/17

\section{RESUMEN}

Objetivo: describir los hallazgos, el nivel de dolor y las complicaciones de la histeroscopia oficinal en el estudio de la paciente infértil.

Materiales y métodos: cohorte retrospectiva de mujeres sometidas a histeroscopia oficinal por historia de falla de implantación, con estudio ecográfico previo normal, entre enero de 2011 y marzo de 2016, en una unidad de fertilidad que atiende pacientes particulares y de medicina prepagada. Se

1 Especialista en Ginecología y Obstetricia; especialista en Reproducción Humana, Unidad de Fertilidad Procreación Médicamente Asistida, Clínica de Marly. Coordinadora de programa de Salud Reproductiva y Humana, Fundación Universitaria Sanitas, Bogotá (Colombia).

2 Especialista en Ginecología y Obstetricia; especialista en Salud Reproductiva y Humana, Fundación Universitaria Sanitas, Bogotá (Colombia).angieuscategui80@hotmail.com

3 Especialista en Ginecología y Obstetricia; especialista en Reproducción Humana, Unidad de Fertilidad Procreación Médicamente Asistida, Clínica de Marly. Programa de Salud Reproductiva y Humana, Fundación Universitaria Sanitas, Bogotá (Colombia).

4 Especialista en Ginecología y Obstetricia; especialista en Reproducción Humana, Bogotá (Colombia).

5 Especialista en Ginecología y Obstetricia, Unidad de Infertilidad y Endocrinología Reproductiva, Unidad de Fertilidad Procreación Médicamente Asistida, Clínica de Marly. Coordinador Departamento de Ginecología, Clínica de Marly, Bogotá (Colombia). excluyeron las pacientes que no continuaron seguimiento en esta institución. Muestreo consecutivo. Se evaluaron variables sociodemográficas, clínicas, hallazgos intraoperatorios, causas de cancelación, nivel de dolor, complicaciones intraoperatorias o posoperatorias. Se realizó análisis descriptivo.

Resultados: de un total de 195 pacientes que cumplieron los criterios de selección se incluyeron 171. Se excluyeron 24 pacientes que no terminaron su tratamiento en esta Unidad. Las pacientes incluidas tenían una mediana de edad de 40 años (RIQ 36-43 años); de ellas, el 86\% nulíparas, y $42 \%$ con diagnóstico de infertilidad primaria, 39\% secundaria y $20 \%$ terciaria. En el $59 \%$ de los casos se encontraron alteraciones endometriales. En el 7,6\% se encontró más de un hallazgo patológico. Hubo una frecuencia de complicaciones de $3 \%$ (dolor y efecto vasovagal), sin complicaciones mayores, y una frecuencia de cancelación de $8 \%$.

Conclusiones: la HSC oficinal con abordaje vaginoscópico es un procedimiento seguro, con buena tolerancia, seguridad y con bajo riesgo de complicaciones a corto y largo plazo. 
Palabras clave: histeroscopia, endoscopia uterina, atención ambulatoria, diagnóstico.

\section{ABSTRACT}

Objective: To describe the findings, level of pain and complications of office hysteroscopy in the workup of the infertile patient.

Materials and methods: Retrospective cohort of women subjected to office hysteroscopy because of a history of implantation failure, with prior ultrasound scan, seen between January 2011 and March 2016 in a fertility unit that serves private patients under the pre-paid medicine regime. Patients who did not come back for follow-up at the institution were excluded. Consecutive sampling was used. Sociodemographic, clinical, intra-operative findings, reasons for cancellation, pain level, and intra-operative or post-operative complications were the variables evaluated. A descriptive analysis was performed.

Results: Of a total of 195 patients who met the selection criteria, 171 were included; 24 patients who did not complete treatment in this Unit were excluded. The mean age of the patients included was 40 years (IQR 36-43 years); $86 \%$ were nulliparous; $42 \%$ had a diagnosis of primary infertility, 39\% secondary infertility, and $20 \%$ tertiary infertility. Endometrial abnormalities were found in $59 \%$ of the cases. There was more than one pathologic finding in $7.6 \%$ of cases. There was a $3 \%$ frequency of complications (pain and vasovagal effect), with no major complications, as well as an $8 \%$ frequency of cancellations.

Conclusions: Office hysteroscopy through the vaginal approach is a safe, well tolerated procedure, associated with a low risk of short and long-term complications.

Key words: Hysteroscopy, uterine endoscopy, outpatient care, diagnosis.

\section{INTRODUCCIÓN}

La patología intracavitaria uterina es frecuente en la edad fértil, se encuentra asociada a patología en- dometrial benigna, como los pólipos, adherencias, hiperplasia, endometritis, resultado de las anomalías mullerianas o por miomatosis uterina submucosa; todas ellas pueden tener un efecto negativo en las tasas de embarazo (1).

Se ha descrito que en la paciente candidata para fertilización in vitro (FIV), la realización inicial de histeroscopia (HSC) mejora de manera significativa la tasa de embarazo, al diagnosticar y tratar la patología endocavitaria, además de aumentar la tasa de éxito de la FIV, exista o no una enfermedad tratada, esto debido a injuria endometrial que genera un proceso inflamatorio local que al parecer aumenta las tasas de embarazo en los grupos que llevan a cabo el procedimiento (2). La HSC, como todo procedimiento que requiere instrumentación del útero, puede estar asociada a dolor, incomodidad y molestia. El dolor se ha asociado con la técnica empleada, la ausencia de parto previo, la experiencia del operador, el tipo de abordaje, el grado de ansiedad de la paciente, el tipo de equipo utilizado, y la necesidad de hacer o no procedimientos operatorios en el mismo acto (3).

Respecto al equipo, los instrumentos de pequeño calibre producen, en teoría, menos dolor, por lo que se requieren menos anestésicos locales o generales. Estos se ofrecen bajo la modalidad de HSC oficinal, como una alternativa para el manejo de la paciente infértil en el consultorio. Se ha descrito como una herramienta útil, poco invasiva, rápida y segura para visualizar de forma directa la cavidad endometrial y el canal endocervical, por medio de la distensión vaginal con solución salina a baja presión $(4,5)$, con bajo riesgo de complicaciones y con menores costos que la técnica de HSC convencional (4). Esta tecnología permite la toma de biopsias dirigidas y la realización de procedimientos quirúrgicos, tales como: polipectomía, algunas miomectomías, resección de tabiques y lisis de adherencias intrauterinas (5).

A pesar de que la recomendación general es la no utilización de anestesia durante la HSC oficinal (6) este aspecto es motivo de controversia ya que 
la dilatación de cérvix con instrumentos delgados como el histerómetro, o la colocación del dispositivo intrauterino frecuentemente se acompañan de dolor (7), con el inconveniente de que este puede tener una repercusión negativa sobre la capacidad de la paciente para cooperar y, por tanto, limita la posibilidad de completar la exploración (6).

Son pocos los estudios en nuestro medio acerca de la seguridad y la factibilidad de llevar a cabo la HSC oficinal por el dolor, y dado que este puede variar por aspectos culturales (8), desarrollamos este estudio con el objetivo de describir las características de la población estudiada, los hallazgos, la frecuencia de complicaciones y el nivel de dolor que experimentaron nuestras pacientes.

\section{MATERIALES Y MÉTODOS}

Cohorte descriptiva retrospectiva, constituida por pacientes en quienes se realizó HSC oficinal por historia de falla de implantación, con estudio ecográfico previo normal, y que fueron llevadas a Terapia de Reproducción asistida en la unidad de Procreación Medicamente Asistida (PMA) entre enero de 2011 a marzo de 2016. Este es un centro privado para el tratamiento de la infertilidad, que atiende pacientes particulares y adscritos a seguros privados de salud, ubicado en Bogotá. Se excluyeron las pacientes que no continuaron seguimiento en esta institución. Se realizó muestreo consecutivo.

Procedimiento. Se identificaron los registros de la historia clínica de las pacientes a quienes se realizó el procedimiento. Una vez identificada la paciente, se revisó la historia clínica a la que se aplicó un formulario para registro de las variables sociodemográficas y clínicas. La HSC se hizo de forma vaginoscópica, con equipo de Bettochi marca Karl Storz; camisa de flujo continuo de $5 \mathrm{~mm}$, con canal operatorio 5 french (Fr); endoscopio de 2,9 mm de diámetro, $30 \mathrm{~cm}$ de longitud y con óptica de 30 , la cual fue realizada por 1 de los 3 miembros que hacen parte del equipo humano especializado de PMA. Se tomó biopsia de endometrio cuando se sospechó patología endometrial (9).
La medición del dolor se hizo mediante el instrumento escala visual análoga (EVA), que se aplicó a todas las pacientes una vez finalizado el procedimiento por parte del especialista; la escala mide el dolor percibido durante la realización de la HSC. Además, se registraron las complicaciones intraoperatorias. Las pacientes fueron citadas a los 8 días para observar complicaciones posoperatorias, y se hizo una llamada al mes del procedimiento.

Se midieron como variables independientes: edad, paridad, tipo de infertilidad (primaria, secundaria o terciaria, que se define cuando alguno de los dos miembros de la pareja ha tenido hijos previamente con otra pareja). Hallazgos en la HSC: pólipos, sinequias, hiperplasia, anomalía mulleriana, mioma y procedimientos operatorios: polipectomía, miomectomía, resección de septo, colocación de Essure $\AA^{\circledR}$ para manejo de hidrosalpinx. Como variables resultado: complicaciones intraoperatorias: desgarro, perforación, reacción vasovagal, sangrado. El nivel de dolor, que se clasificó como: menor a 4 (ausente o leve) entre 4-7 (moderado), mayor de 7 (severo) (10). Suspensión del procedimiento y la causa de cancelación. Las complicaciones intra y posoperatorias se clasificaron en mayores y menores, las mayores son aquellas que requieren hospitalización para su control y manejo, y las menores no ponen en peligro la vida del individuo, pero se asocian a incomodidad y disconfort (10). Los resultados se presentan en frecuencias absolutas y relativas para las variables cualitativas, y en medianas y rangos intercuartílicos para las variables cuantitativas.

Aspectos éticos. El estudio fue aprobado previamente por el comité de ética en investigación de la Fundación Universitaria Sanitas.

\section{RESULTADOS}

De un total de 195 pacientes que cumplieron los criterios de selección se incluyeron 171 pacientes después de aplicar los criterios de exclusión.

En cuanto a las características basales se encontró para la edad una mediana de 40 años (RIQ 36-43 
años), siendo 147 (86,0\%) nulíparas, 21 de ellas (12,3\%) primíparas, y 3 (1,7\%) multíparas. Con respecto al tipo de infertilidad, en 71 (42\%) era primaria, en 66 (39\%) secundaria y en 34 (20\%) terciaria. Durante el procedimiento de HSC oficinal, 70 de ellas (41 \%) no presentaron ninguna alteración en la cavidad. En 101 (59\%) pacientes se encontró patología intracavitaria; el hallazgo más frecuente fue el pólipo en 40 (39,6\%), seguido de endometritis crónica en 26 (25,7\%). En 8 (7,6\%) pacientes se encontró más de un hallazgo, estos incluían diferentes combinaciones de pólipos, sinequias, endometritis crónica, miomas submucosos, anomalías mullerianas (tabla 1). Se realizaron procedimientos intraoperatorios en 38 (22\%) pacientes, de estas se realizó adhesiolisis en 12 (32\%), resección de pólipo en 22 (59\%), resección de septo en 4 (11\%). En tres de las anteriores pacientes se colocó Essure® (8\%). A 94 de las mujeres incluidas (55\%) se les tomó biopsia durante la HSC, reportándose pólipo en 41 (44\%); esta dio como resultado: endometrio sin alteraciones

\begin{tabular}{|l|l|}
\hline \multicolumn{2}{|c|}{ Tabla 1. } \\
$\begin{array}{r}\text { Hallazgos durante la histeroscopia oficinal } \\
\text { en mujeres con infertilidad en un centro } \\
\text { privado de Bogotá, 2011-2016\% }\end{array}$ \\
\hline \multicolumn{1}{|c|}{ Hallazgo } & n = 171 (\%) \\
\hline Cavidad sin alteración & $70(41 \%)$ \\
\hline Pólipo & $40(23,4 \%)$ \\
\hline Sinequia uterina & $14(8,2 \%)$ \\
\hline Sinequia cervical & $9(5,3 \%)$ \\
\hline Endometritis crónica & $26(15,2 \%)$ \\
\hline Mioma submucoso tipo 0 & $3(1,7 \%)$ \\
\hline Mioma submucoso tipo 1 & $1(0,6 \%)$ \\
\hline Mioma submucoso tipo 2 & $4(2,3 \%)$ \\
\hline Anomalía mulleriana & $14(8,2 \%)$ \\
\hline Engrosamiento endometrial & $5(3,0 \%)$ \\
\hline Atrofia endometrial & $1(0,6 \%)$ \\
\hline
\end{tabular}

* Del total de pacientes se identificaron hallazgos anormales en 101. patológicas en 36 (38\%), endometritis crónica en 12 (13\%) e hiperplasia simple en 5 casos (5\%). No hubo complicaciones mayores; se presentaron complicaciones menores en 5 pacientes ( $3 \%$ ), dadas por dolor severo que requirió suspender el procedimiento en 2 pacientes $(1,2 \%)$ y el efecto vasovagal en 3 pacientes $(1,8 \%)$, a las que presentaron efecto vagal se les terminó el procedimiento una vez manejado.

Adicionalmente, hubo 13 cancelaciones (8\%); de estas, $80 \%$ (n:11) por necesidad de procedimiento en quirófano o por intolerancia al dolor (1,2 \% (n:2). La distribución del nivel de dolor se presenta en la tabla 2.

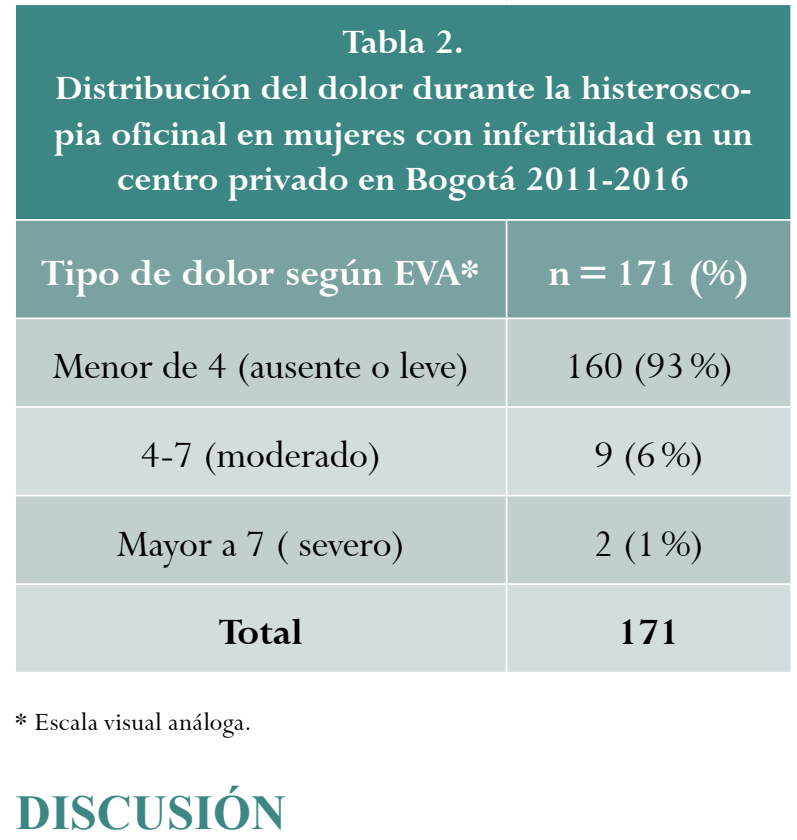

Este estudio mostró que la HSC oficinal es un procedimiento factible, que se pudo realizar al menos en el $92 \%$ de las pacientes, con una frecuencia de complicaciones del 3\%, todas ellas menores. Además, se encontró una frecuencia del 59\% de anomalías intracavitarias.

Con respecto a factibilidad y frecuencia de complicaciones se tienen datos de otros países como el estudio de Cincinelli con 6.000 paciente que informa que con el histeroscopio de $5 \mathrm{~mm}$ el procedimiento fue satisfactorio en el $92 \%$ de las pacientes, con promedio de dolor de 1,09\% ( \pm $0,53)$ y efecto vagal de 2,2\% (11). Darwish (12), 
en un ensayo controlado randomizado que incluía 156 pacientes infértiles llevadas a HSC con técnica vaginoscópica reporta una tolerancia del $100 \%$, y el dolor fue calificado como leve o ausente en el $94 \%$ de los casos. Bettochi et al. (13), en 11.000 pacientes llevadas a HSC con técnica oficinal, informan que cerca del 99\% no reportan disconfort relacionado con el procedimiento. Campo et al. (14) también realizaron un estudio aleatorizado controlado con 240 pacientes y una media de dolor en EVA de 1,8. Por otra parte, otros autores informan una frecuencia de dolor superior a la encontrada por nosotros. Readman (15) en una revisión sistemática que incluyó 29 estudios y 10.232 pacientes, reporta que el éxito puede variar entre 69 a $100 \%$, y la falla se debe al dolor en un 29,4\% de los casos, probablemente porque recibieron una analgesia inadecuada. Cincinelli (16) en 2007, reportó una incidencia de dolor moderado a intenso de $22 \%$ en su serie. En una serie con 1.028 pacientes, van Kerkvoorde reportó tasas mucho más altas, en las que el $75 \%$ presentó dolor, y el $14 \%$ efecto vasovagal (17). De Caravalho (6) reportó que 34,8\% de las pacientes a las que se les realizó el estudio reportaron algún tipo de dolor, y fue esta la principal causa de cancelación; como se observa, este aspecto sigue siendo un tema de debate. Estas diferencias pueden estar relacionadas con el método de distención, la experiencia del operador, la preparación de la paciente, limitaciones en la movilidad uterina (18) que deben tenerse en cuenta en el análisis de los estudios.

Nuestros hallazgos respecto a las complicaciones son similares a otros estudios, en el estudio realizado por Carabias y cols en Chile da cuenta de 2,6\% (19) Bakas et al. (20) reportan una frecuencia de complicaciones de 1,3\% (4 de 240) donde fue el efecto vasovagal la principal, estos autores no informan laceraciones, sangrado o perforación uterina.

Respecto a la frecuencia de hallazgos anormales intracavitarios nuestros resultados son ligeramente superiores al índice de anomalías reportadas por Ferring et al. cuando se realiza una HSC antes de la
FIV (21). Los diagnósticos son similares a la serie de Nagele (22), que informa 2.500 histeroscopias, en donde los hallazgos más comunes fueron los miomas (36\%) y los pólipos endometriales (20\%). En el estudio latinoamericano de Carabias mencionado (19), el pólipo fue el hallazgo más frecuente (27\%), seguido por mioma submucoso $10 \%$.

Como debilidad se encuentra la exclusión, por no seguir en la institución, de un total de 24 pacientes (12\%) y 13 pacientes ( $8 \%$ ) en las que no se pudo realizar el procedimiento, lo que pudo afectar la estimación de la frecuencia de dolor; también la naturaleza retrospectiva del estudio y las limitaciones para la generalización.

Nuestro estudio sugiere que la HSC oficinal es un procedimiento útil. A futuro sería pertinente evaluar los resultados de fertilidad tras su realización, y establecer la relación entre resultados de tratamiento de fertilidad y manejo de patologías endocavitarias específicas. También es importante determinar requerimientos anestésicos (13) y, si se requiere, la preparación cervical (23).

\section{CONCLUSIONES}

La HSC oficinal con abordaje vaginoscópico es un procedimiento seguro, con buena tolerancia, seguridad y con bajo riesgo de complicaciones a corto y largo plazo. Sin embargo, aunque en la mayoría de las pacientes es muy bien tolerado, es necesaria una selección adecuada para minimizar el disconfort y disminuir los niveles de ansiedad. Se requieren estudios con grupo control aleatorizados y resultados a largo plazo en infertilidad para evaluar la efectividad de esta tecnología.

\section{REFERENCIAS}

1. Valle RF. Hysteroscopy in the evaluation of female infertility. Am J Obstet Gynecol. 1980;137:425-31. https://doi.org/10.1016/0002-9378(80)91122-9.

2. Almog B, Shalom-Paz E, Dufort D, Tulandi T. Promoting implantation by local injury to the endometrium. Fertil Steril. 2010;94:2026-9. https://doi. org/10.1016/j.fertnstert.2009.12.075. 
3. Cooper NAM, Smith P, Khan KS, Clark TJ. A systematic review of the effect of the distension medium on pain during outpatient hysteroscopy. Fertil Steril. 2011;95:264-71. https://doi.org/10.1016/j.fertnstert.2010.04.080.

4. Bettocchi S, Selvaggi L. A vaginoscopic approach to reduce the pain of office hysteroscopy. J Am Assoc Gynecol Laparosc. 1997;4:255-8. https://doi. org/10.1016/S1074-3804(97)80019-9.

5. Di Spiezio Sardo A, Taylor A, Tsirkas P, Mastrogamvrakis G, Sharma M, Magos A. Hysteroscopy: a technique for all? Analysis of 5,000 outpatient hysteroscopies. Fertil Steril. 2008;89:438-43. https:/doi. org/10.1016/j.fertnstert.2007.02.056.

6. de Carvalho Schettini JA, Ramos de Amorim MM, Ribeiro Costa AA, Albuquerque Neto LC. Pain evaluation in outpatients undergoing diagnostic anesthesia-free hysteroscopy in a teaching hospital: A cohort study. J Minim Invasive Gynecol. 2007;14:729-35. https:// doi.org/10.1016/j.jmig.2007.05.009

7. Anguzu R, Tweheyo R, Sekandi JN, Zalwango V, Muhumuza C, Tusiime S, et al. Knowledge and attitudes towards use of long acting reversible contraceptives among women of reproductive age in Lubaga division, Kampala district, Uganda. BMC Res Notes. 2014;7:153. https:// doi.org/10.1186/1756-0500-7-153.

8. Bustos Domínguez R. Elementos para una antropología del dolor: el aporte de David Le Breton. Acta Bioeth. 2000;6:103-11. https://doi.org/10.4067/ S1726-569X2000000100008.

9. Valle RF. Hysteroscopic evaluation of patients with abnormal uterine bleeding. Surg Gynecol Obstet. 1981;153:521-6.

10. Serrano MS, Caballero J, Ca-as A, García-Saura PL, Serrano -Álvarez C, Prieto J, et al. Valoración del dolor (I). Rev Soc Esp Dolor. 2002;9:94-108.

11. Cicinelli E, Parisi C, Galantino P, Pinto V, Barba B, Schonauer S. Reliability, feasibility, and safety of minihysteroscopy with a vaginoscopic approach: Experience with 6,000 cases. Fertil Steril. 2003;80:199-202. https://doi.org/10.1016/S0015-0282(03)00546-6.

12. Darwish AM, Hassanin AI, Aleem MAA, Mohammad II, Aboushama IH. Routine vaginoscopic office hyster- oscopy in modern infertility work-up: A randomized controlled trial. Gynecol Surg. 2014;11:185-9. https:// doi.org/10.1007/s10397-014-0840-x.

13. Bettocchi S, Nappi L, Ceci O, Selvaggi L. Office hysteroscopy. Obstet Gynecol Clin North Am. 2004;31:64154, xi.

14. Campo R, van Belle Y, Rombauts L, Brosens I, Gordts S. Office mini-hysteroscopy. Hum Reprodn Update. 1999;5:73-81. https://doi.org/10.1093/hu$\operatorname{mupd} / 5.1 .73$.

15. Readman E, Maher PJ. Pain relief and outpatient hysteroscopy: A literature review. J Am Assoc Gynecol Laparosc. 2004;11:315-9. https://doi.org/10.1016/ S1074-3804(05)60042-4.

16. Cicinelli E, Rossi AC, Marinaccio M, Matteo M, Saliani N, Tinelli R. Predictive factors for pain experienced at office fluid minihysteroscopy. J Minim Invasive Gynecol. 2007;14:485-8. https://doi.org/10.1016/j. jmig.2012.03.003.

17. van Kerkvoorde TC, Veersema S, Timmermans A. Long-Term Complications of Office Hysteroscopy: Analysis of 1028 Cases. J Minim Invasive Gynecol. 2012;19:494-7. https://doi.org/10.1016/j. jmig.2012.03.003.

18. Assessment T. Technology assessment No. 7: Hysteroscopy. Obstet Gynecol. 2011;117:1486-91. https:// doi.org/10.1097/AOG.0b013e3182238c7d.

19. Carabias P, Celades-Filella M, Zapardiel I, AlsinaMaqueda A, Genover-Llimona E. Experience and results of office hysteroscopy at a primary hospital. J Obstet Gynaecol. 2014;34:54-6. https://doi.org/10.3 109/01443615.2013.782277.

20. Bakas P, Hassiakos D, Grigoriadis C, Vlahos N, Liapis A, Gregoriou O. Role of hysteroscopy prior to assisted reproduction techniques. J Minim Invasive Gynecol. 2014;21:233-7. https://doi.org/10.1016/j. jmig.2013.07.023.

21. Ferring AG, Baird DT, Collins J, Cooke I, Cohen J, Evers JLH, et al. Optimal use of infertility diagnostic tests and treatments. Hum Reprod. 2000;15:723-32. https://doi.org/10.1093/humrep/15.3.723.

22. Nagele F, O'Connor H, Davies A, Badawy A, Mohamed H, Magos A. 2500 Outpatient diagnostic hysteros- 
copies. Obstet Gynecol. 1996;88:87-92.https://doi. org/10.1016/0029-7844(96)00108-1.

23. Campo R, Molinas CR, Rombauts L, Mestdagh G, Lauwers M, Braekmans P, et al. Prospective multi- centre randomized controlled trial to evaluate factors influencing the success rate of office diagnostic hysteroscopy. Hum Reprod. 2005;20:258-63. https:// doi.org/10.1093/humrep/deh559. 